\title{
Ultrasound Imaging of Pipeline Crack Based on Composite Transducer Array
}

CrossMark

\author{
Shou-Peng Song ${ }^{*}$ and Ying-Jie $\mathrm{Ni}$
}

\begin{abstract}
Cracks, especially small cracks are difficult to be detected in oil and gas transportation pipelines buried underground or covered with layers of material by using the traditional ultrasonic inspection techniques. Therefore, a new composite ultrasonic transducer array with three acoustic beam incidence modes is developed. The space model of the array is also established to obtain the defect reflection point location. And the crack ultrasound image is thus formed through a series of small cubical elements expanded around the point locations by using the projection of binarization values extracted from the received ultrasonic echo signals. Laboratory experiments are performed on a pipeline sample with different types of cracks to verify the effectiveness and performance of the proposed technique. From the image, the presence of small cracks can be clearly observed, in addition to the sizes and orientations of the cracks. The proposed technique can not only inspect common flaws, but also detect cracks with various orientations, which is helpful for defect evaluation in pipeline testing.
\end{abstract}

Keywords: Composite transducer array, Ultrasound imaging, Crack, NDT\&E

\section{Introduction}

Pipelines constitute a crucial means for transporting oil and gas. Pipeline cracks lead to leakage accidents and cause considerable losses [1-4]. Therefore, the initial detection of cracks must be timely and effective. Currently, the magnetic flux leakage inspection method and ultrasonic inspection method are commonly used to test pipelines $[5,6]$. Because ultrasonic inspection technology is highly efficient, and because it detects more types of defects than other methods do, it has been developed rapidly in recent years.

Most existing industrial tools that are placed inside pipelines for ultrasonic inspection adopt acoustic beams propagating at normal incidence, which is suitable for inspecting the flaws caused by changes in the pipe wall thickness. However, these methods are insensitive to cracks, particularly small cracks. In light of this shortcoming, numerous ultrasonic inspection tools have been developed for improving inspection performance. For

*Correspondence: songshoupeng@126.com

Department of Instrument Science and Engineering, School

of Mechanical Engineering, Jiangsu University, Zhenjiang 212013, China example, GE has developed a tool, called UltraScan Duo, with an ultrasonic phased array transducer for inspecting defects in pipe walls, including cracks and defects caused by changes of thickness [7]. The UltraScan Duo can detect cracks that are at least $1 \mathrm{~mm}$ deep and $25 \mathrm{~mm}$ long $[8,9]$. However, the UltraScan Duo requires a CPU to control its phased-beam forming accurately to obtain the best beam characteristics, which complicates matters. A pipeline inspection tool from the German company ROSEN uses an electromagnetic acoustic transducer (EMAT); this tool is named RoCD EMAT-C. It is specialized for pipeline crack and anticorrosive coating inspection; it can detect cracks that are at least $1 \mathrm{~mm}$ deep and $40 \mathrm{~mm}$ long [9-12]. However, its sensitivity level is lower than those of other ultrasonic inspection methods. Another ultrasonic pipeline crack inspection tool, called EVO Series UC 1.0, developed by the German NDT Global Company, can detect cracks that are at least $1 \mathrm{~mm}$ deep and $40 \mathrm{~mm}$ long [13-15]. However, the transducers on the EVO Series UC 1.0 are mounted on a pigging tool and operate at a circumferential angle of incidence, which is limited to the detection of transversely oriented cracks. 
Methods for visual description of pipeline defects include B-scan ultrasound imaging, C-scan ultrasound imaging [16, 17], and ultrasonic phased array imaging [18-20]. A B-scan image reflects the distribution of defects on a pipeline's longitudinal section, and a C-scan image reflects the distribution of defects on a pipeline's cylindrical surface. A phased array image is a synthetic result of C-scan imaging conducted at acoustic beam incidence angles; analysis of a phased array image can yield more information than analysis of a C-scan image. However, the high cost of equipment and inspection restricts its field applications. In addition, these imaging methods display only two-dimensional information regarding defects rather than three-dimensional information [21], and two-dimensional information cannot meet the requirements of an accurate quantitative analysis of the spatial distribution of defects.

To address these problems, this paper presents a new composite transducer array for ultrasonic inspection of pipelines. This composite array can scan defects from different acoustic beam incidence angles by combining normal incidence, axial oblique incidence, and circumferential oblique incidence modes, which improves its sensitivity for crack detection. In addition, the proposed ultrasound imaging method provides three-dimensional information regarding defects, which is convenient for quantization and evaluation of the defects.

\section{Establishment of Composite Ultrasonic Transducer Array Space Model}

The composite transducer array contains three acoustic beam incidence modes, namely normal incidence, axial oblique incidence, and circumferential oblique incidence. The normal incidence mode is used to detect flaws caused by changes in pipe wall thickness; the axial oblique incidence mode is used to detect circumferentially oriented cracks; and the circumferential oblique incidence mode is used to detect axially oriented cracks. The combination of the three incidence modes yields a defect reflecting surface that improves the detect ability of defects at different orientations. These three incidence modes can also provide reflector information of different dimensions for the same defect. This method can scan the whole pipe wall through each acoustic beam incidence mode when the transducer array passes through the pipeline. The number of transducers used for each incidence mode is determined by the pipeline diameter and transducer parameters. Figure 1 shows the geometric structure of the composite transducer array.

The composite array includes two rings of transducers operating in normal incidence mode, marked as

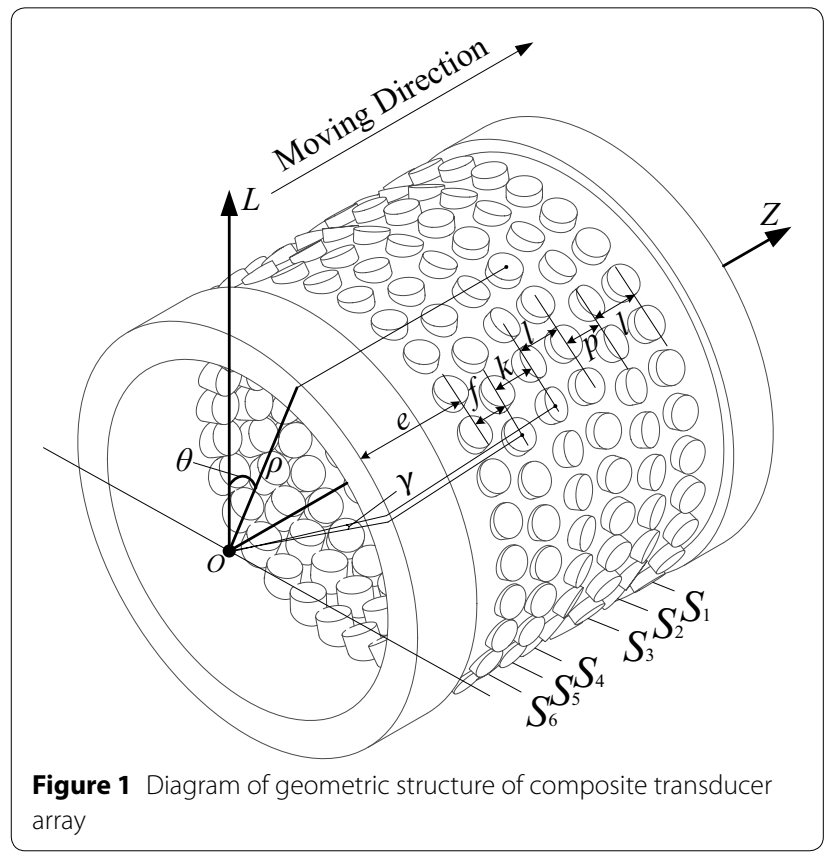

$S_{1}$ and $S_{3}$; two rings of transducers operating in axial oblique incidence mode, marked as $S_{2}$ and $S_{4}$; and two rings of transducers operating in circumferential oblique incidence mode, marked as $S_{5}$ and $S_{6}$. The order of the transducer rings along with the direction of array motion is $S_{6} \rightarrow S_{5} \rightarrow S_{4} \rightarrow S_{3} \rightarrow S_{2} \rightarrow S_{1}$. Transducers are evenly distributed in each ring. The acoustic beam incidence angles of the oblique incidence modes are all $\alpha$, which is selected between the first critical angle and the second critical angle for the water-steel interface to ensure the use of a pure ultrasonic shear wave for defect inspection (water is applied as couplant in testing).

A cylindrical coordinate system $(\rho \theta z)$ on the composite array is established with the origin point $O$ selected at the axial center of the rear face on the composite array supporter, where $\rho$ is the radial distance between the geometric center of the transducer and the axis of the composite array. $\theta$ indicates the circumferential angle between the radial line of transducers and the vertical line $L$, with the counterclockwise direction being considered as the positive direction. The $z$-axis coincides with the pipeline axis, with the direction of array motion being considered as the positive direction. The transducer liftoff on the $m$ th ring is marked as $h_{m}$ with $h_{1}=h_{3}, h_{2}=h_{4}$, $h_{5}=h_{6}$. Considering the acoustic beam interference between the transducer and its housing shell, and the transducer beam covering the whole pipe wall, the lift-off of $h_{1}$ and $h_{2}$ must satisfy the following equation: 


$$
\frac{d}{2} \sin \alpha+h_{2} \cos \alpha>h_{1}>\frac{d}{2} \sin \alpha+h_{2} \cos \alpha+\frac{\frac{d}{2}(1+\cos \alpha)-l}{\tan (\alpha+\varphi)}
$$

where $d$ is the transducer diameter, $\phi$ is the half-spread angle of the transducer acoustic beam, $l$ is the axial distance between the geometric center of the outer surface of the transducer carrier hole on two neighboring rings.

A transducer in $S_{1}$ is numbered as 1 , and it is marked as $C_{11}$. A transducer in $S_{2}$ with the minimum included angle to $C_{11}$ is marked as $C_{21}$. Analogously, the transducer in the $m$ th ring of the composite array numbered $n$ is marked as $C_{m n}$. Define the coordinate of an arbitrary transducer in the cylindrical coordinate system as $\boldsymbol{C}_{\boldsymbol{m} \boldsymbol{n}}=\left[\begin{array}{lll}\rho_{m n} & \theta_{m n} & z_{m n}\end{array}\right]$. The geometrical structural diagrams of the normal incidence transducer array, axial oblique incidence transducer array, and circumferential oblique incidence transducer array are shown in Figures 2, 3 and 4, respectively. According to the geometrical relationship, the coordinate of an arbitrary transducer in the composite array can be expressed as follows:

$$
C_{\boldsymbol{m} n}=\left[\sqrt{R^{2}-2 R h_{m} \cos \eta+h_{m}^{2}} \frac{4 \pi n+\pi N}{2 N}-G \varepsilon e+\sum_{i=1}^{I} f_{i} \varepsilon_{i}(x)\right]
$$

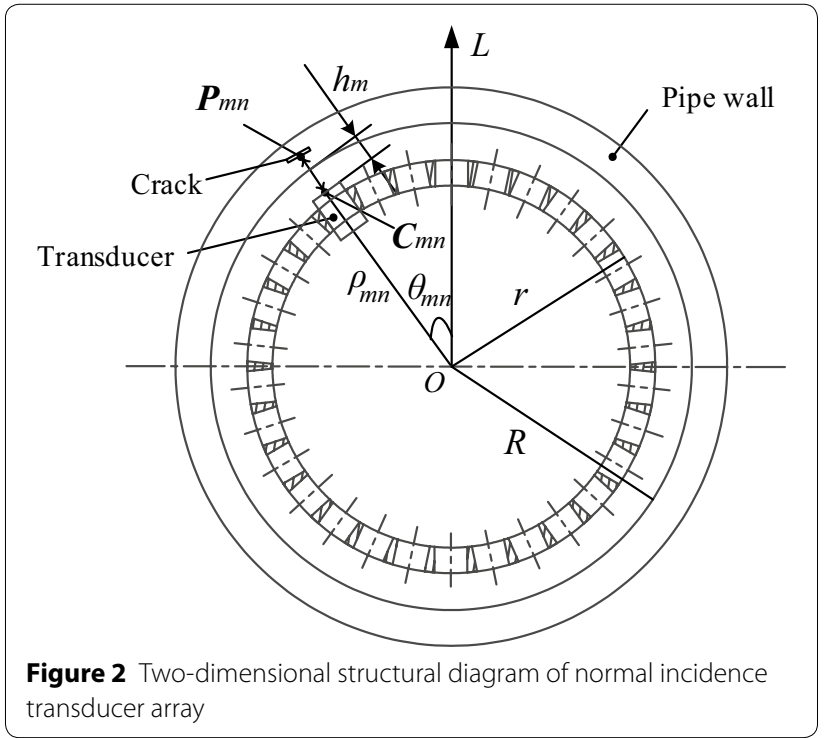

where $M$ is the number of rings, $N$ is the number of transducers in each ring, $R$ is the inner radius of the pipeline, $r$ is the outer radius of the transducer carrier, $e$ is the axial distance between the rear end surface of the transducer carrier and the geometric center of the outer surface of the transducer carrier holes in $S_{6}, f$ is the axial distance between the geometric centers of $S_{5}$ and $S_{6}, k$ is the axial distance between the geometric centers of geometric centers of $S_{4}$ and $S_{5}, p$ is the axial distance between the geometric centers of geometric centers of $S_{2}$ and $S_{3}, \gamma$ is the circumferential angle of the geometric center of two adjacent transducer carrier holes in $S_{4}$ and $S_{5}$.

$$
\begin{aligned}
& m=1,2,3, \ldots, M, \\
& n=1,2,3, \ldots, N, \\
& \eta=\alpha \varepsilon(b-1),
\end{aligned}
$$

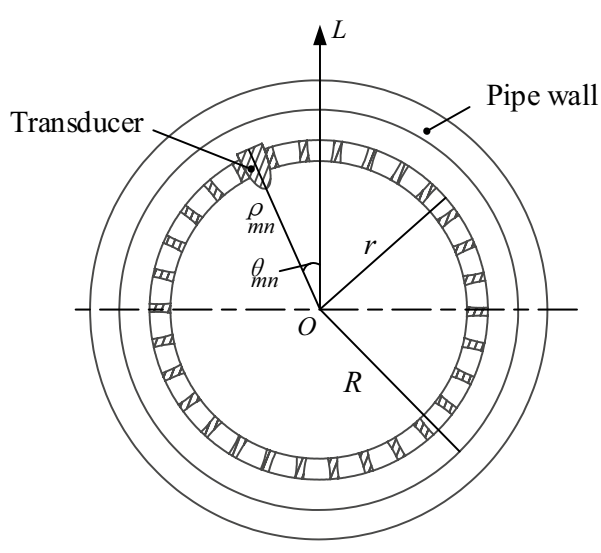

a Transverse section of array

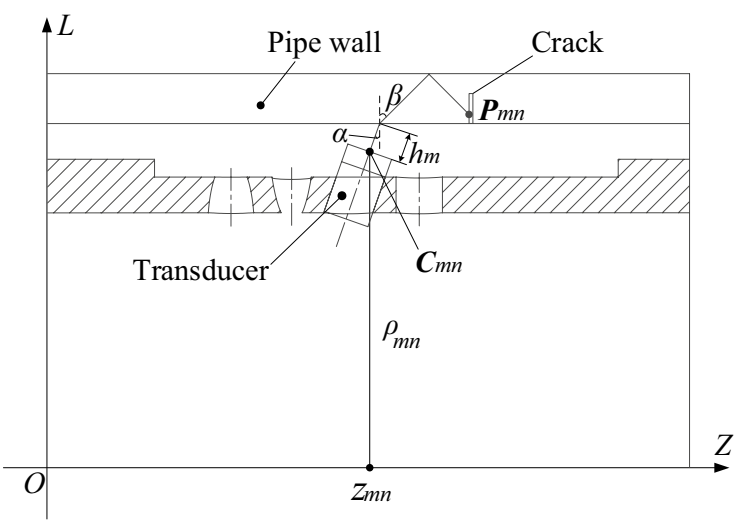

b Longitudinal section of array

Figure 3 Two-dimensional structural diagrams of axial oblique incidence transducer array 


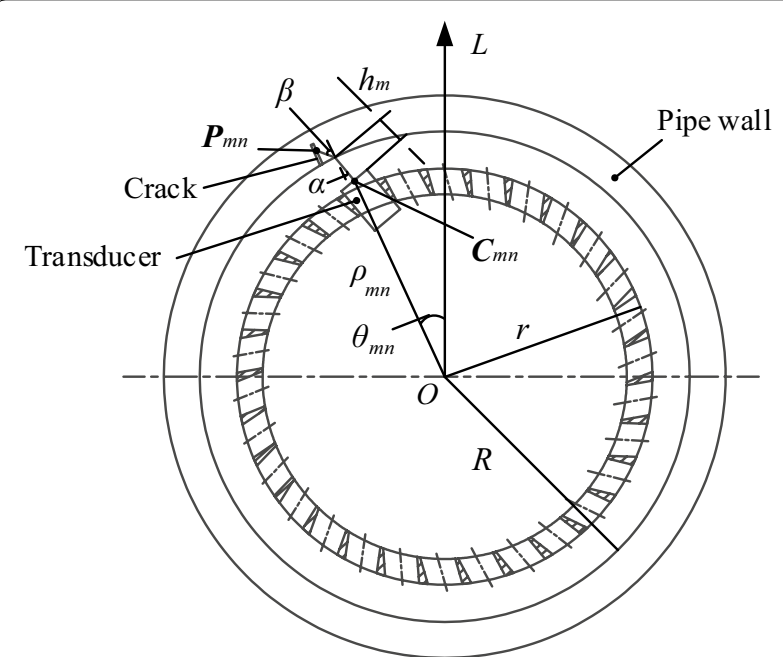

Figure 4 Two-dimensional structural diagram of circumferential oblique incidence transducer array

$\varepsilon(x)= \begin{cases}1, & x>0 \\ 0, & x \leqslant 0\end{cases}$

$b= \begin{cases}1, & \text { Normal incidence } \\ 2, & \text { Axial oblique incidence } \\ 3, & \text { Circumferential oblique incidence. }\end{cases}$

$\boldsymbol{G}=\left[\gamma \frac{\pi+2 \pi N_{m}}{N_{m}} 2 \pi-\delta\right]$

$\boldsymbol{\varepsilon}=\left[\begin{array}{c}\varepsilon(5-m) \\ \varepsilon(m-2)+\varepsilon(m-5) \\ \varepsilon(m-4)\end{array}\right]$

$\delta=\cos ^{-1} \frac{r^{2}+\rho^{2}-\left(c-h_{m}\right)^{2}}{2 r \rho}$

$c=\frac{r \sin \left(\pi-\alpha-\sin ^{-1} \frac{R \sin \alpha}{r}\right)}{\sin \alpha}$

$I=M-1$,

$$
\begin{aligned}
& \varepsilon_{i}(x)=\varepsilon(i-m+1), \\
& f_{i}= \begin{cases}p+\left(\frac{R-r}{\sin \alpha}-h_{m} \sin \alpha\right), & i=2 ; \\
l-\left(\frac{R-r}{\sin \alpha}-h_{m} \sin \alpha\right), & i=1,3 ; \\
k+\left(\frac{R-r}{\sin \alpha}-h_{m} \sin \alpha\right), & i=4 ; \\
f, \quad i=5 .\end{cases}
\end{aligned}
$$

All the transducer coordinates on the composite array can then form an $M \times N$ matrix $C$, which is called the transducer spatial model, and is expressed as

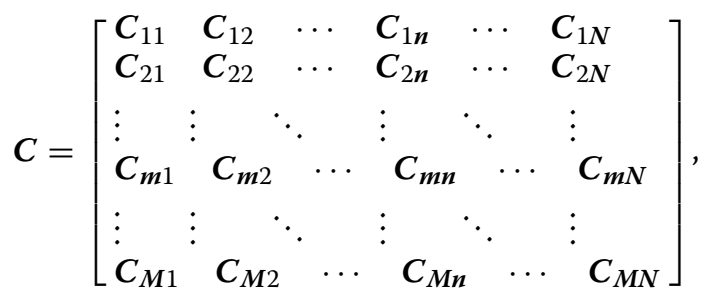

\section{Defect Ultrasound Imaging Method}

In an actual inspection, the axial oblique incidence transducer array applies the single-bounce technique (also called the double traverse technique) [22] to obtain the parameters of the defect reflection signal. The normal incidence and circumferential oblique incidence transducer arrays apply a straight beam incidence method [22] to obtain the parameters of the defect reflection signal. The time of flight of the defect echo can be calculated according to the propagation path of an ultrasonic wave in the pipeline and the transducer space model $\boldsymbol{C}$.

Suppose that $\boldsymbol{P}_{\boldsymbol{m} \boldsymbol{n}}$ represents the spatial location of the reflection point on a defect corresponding to an arbitrary transducer $\boldsymbol{C}_{\boldsymbol{m} \boldsymbol{n}}$ in the composite array. It can be deduced from the spatial distribution of transducers in the composite array and from the geometric acoustic propagation characteristics, which can be expressed as,

$$
\boldsymbol{P}_{\boldsymbol{m} \boldsymbol{n}}^{\mathrm{T}}=\left[\begin{array}{lll}
0 & & \\
& 1 & \\
& & 1
\end{array}\right] \boldsymbol{C}_{\boldsymbol{m} \boldsymbol{n}}^{\mathrm{T}}+\boldsymbol{Q}_{\boldsymbol{m} \boldsymbol{n}}^{\mathrm{T}}
$$

where $\boldsymbol{C}_{\boldsymbol{m} \boldsymbol{n}}^{\mathrm{T}}$ is the transposition of $\boldsymbol{C}_{\boldsymbol{m} \boldsymbol{n}}, \boldsymbol{P}_{\boldsymbol{m} \boldsymbol{n}}^{\mathrm{T}}$ is the transposition of $\boldsymbol{P}_{\boldsymbol{m} \boldsymbol{n}}, \boldsymbol{Q}_{\boldsymbol{m} \boldsymbol{n}}^{\mathrm{T}}$ is the transposition of $\boldsymbol{Q}_{\boldsymbol{m} \boldsymbol{n}}$.

$$
\boldsymbol{Q}_{\boldsymbol{m} \boldsymbol{n}}=\left[R \cos \mu+2 B \sum_{i=1}^{J}(-1)^{i} \varepsilon_{i}(x)+w \cos \sigma \psi \varepsilon(m-4) g \sum_{i=1}^{J}(-1)^{i} \varepsilon_{i}(x)\right] \text {, }
$$


where $\beta$ is the beam refraction angle of the water-steel interface, $B$ is the pipe wall thickness, $v_{w}$ is the ultrasonic velocity in water, $v_{s l}$ is the velocity of ultrasonic shear waves in steel, $v_{s s}$ is the velocity of ultrasonic longitudinal waves in steel, $t_{m n}$ is the time of arrival of the defect echo received by the transducer $\boldsymbol{C}_{\boldsymbol{m} n}$,

$$
\begin{aligned}
\mu & =\left(\frac{\pi}{2}-\xi-\beta\right) \varepsilon(m-4), \\
\xi & =\tan ^{-1} \frac{w+R \cos \beta}{R \sin \beta}, \\
J=\frac{2 M}{3}, \sigma & =\left(\frac{\pi}{2}-\xi\right) \varepsilon(m-4)+(\beta+\pi) \sum_{i=1}^{J}(-1)^{i} \varepsilon_{i}(x), \\
w & =\left\{\begin{array}{l}
\left(\frac{t_{m n}}{2}-\frac{h_{m}}{v_{w}}\right) v_{s l}, \text { normal incidence; } \\
\left(\frac{t_{m n}}{2}-\frac{h_{m}}{v_{w}}\right) v_{s s}, \text { oblique incidence. }
\end{array}\right. \\
\psi & =\sin ^{-1} \frac{h_{m} \sin \alpha}{D}+\sin ^{-1} \frac{w \cos \xi}{R}, \\
g & =h_{m} \sin \alpha+w \sin \beta, \\
D & =\sqrt{\left(R-h_{m} \cos \alpha\right)^{2}+h_{m}^{2} \sin ^{2} \alpha .}
\end{aligned}
$$

The coordinates of each acoustic reflection point on the defect can then be obtained. A matrix $\boldsymbol{P}$ can be assembled by integrating all these coordinates and can be expressed as

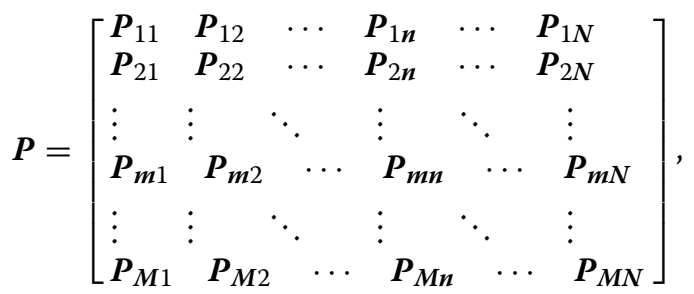

If the corresponding transducer receives an echo signal from a defect, $\boldsymbol{P}_{\boldsymbol{m} \boldsymbol{n}}$ indicates the presence of the defect and the position of the acoustic wave reflection point on the defect. If no signal is received, no defect sexist in the acoustic wave transmission path, and the corresponding $\boldsymbol{P}_{\boldsymbol{m} \boldsymbol{n}}$ is set to zero. The nonzero $\boldsymbol{P}_{\boldsymbol{m} \boldsymbol{n}}$ is applied to expand a small cubical element as the center of a cube. The image of the defect can be obtained by filling the cubical elements with uniform value (presented with the same color in the image). Binarization processing is applied in ultrasound imaging to eliminate noise interference, thus improving the noise stability of the images, and passivate the sensitivity of defects to the angle of incident beams.

\section{Crack Detection Experiments and Imaging on a Pipeline}

To verify the effectiveness of the proposed inspection method, as well as the imaging quality of the composite array, experiments were performed on a pipeline sample with artificial cracks of various orientations and different sizes. Figure 5 shows the experimental platform.

The sample in the experiment was a semi sectional seamless steel pipeline with artificial cracks. Its inside diameter was $191 \mathrm{~mm}$ and pipe wall was $14 \mathrm{~mm}$. Table 1 presents the crack parameters. The transducer in the experiment was a customized immersion straight probe with the material of the sensitive element of PZT, a wafer diameter of $10 \mathrm{~mm}$ and central frequency of $5 \mathrm{MHz}$. The lift-offs of normal incidence mode, axial oblique incidence mode, and circumferential oblique incidence mode were 14,16 and $14 \mathrm{~mm}$, respectively. The ultrasonic transmitting and receiving circuit was a self-made multichannel ultrasonic transceiver. The data acquisition and recording unit was an NI PXI-5152.

The outside diameter of the transducer carrier of the composite array in the experiment was $161 \mathrm{~mm}$. The axial distance between the rear end surface of the transducer carrier and the geometric center of the outer surface of

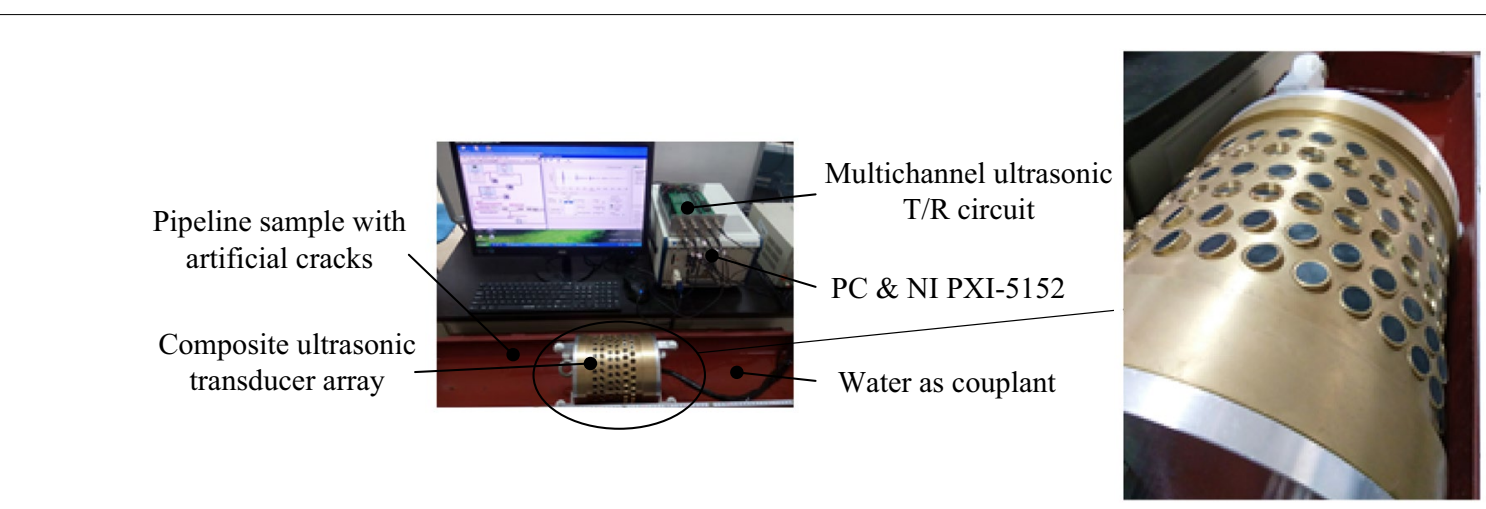

Figure 5 Experimental platform 
Table 1 Crack parameters

\begin{tabular}{lllll}
\hline No. & Crack type & Length $(\mathbf{m m})$ & Width $(\mathbf{m m})$ & Depth $(\mathbf{m m})$ \\
\hline $1^{\#}$ & Internal axial crack & 10 & 1 & 3.6 \\
$2^{\#}$ & External circumferential crack & 10 & 0.5 & 6 \\
$3^{\#}$ & Internal circumferential crack & 10 & 1 & 3.6 \\
\hline
\end{tabular}

the transducer carrier holes in $S_{6}$ was $52 \mathrm{~mm}$. Moreover, the axial distances between the geometric centers of $S_{5}$ and $S_{6}, S_{4}$ and $S_{5}, S_{3}$ and $S_{4}, S_{2}$ and $S_{3}$, and $S_{1}$ and $S_{2}$ were $16,20,19,20$ and $19 \mathrm{~mm}$, respectively. The circumferential angle of the geometric centers of two transducer carrier holes in $S_{4}$ and $S_{5}$ was $3.7^{\circ}$.

In the experiments, the moving step of the composite array was $1 \mathrm{~mm}$. After all reflected signals of the transducers were recorded, the composite array was moved to the next stepping location to start another inspection period. If a crack is presented on the acoustic transmission path, there will be a reflected signal. Otherwise, there will be no reflected signal of the defect. Then, the transducer spatial model was established, and the defect image was obtained according to the proposed method. The volume of the cubical element for imaging was set as $1 \times 1 \times 1 \mathrm{~mm}^{3}$.

\subsection{First Crack}

The first crack was an axial crack, which was manufactured on the internal surface of the pipeline sample. In the experiment, only one transducer in the composite array received defect echoes repeatedly at the different stepping positions. Figure 6 shows one of the echo signals. Figure 7(a) depicts a picture of the first crack, and Figure 7(b) and (c) present images of the first crack obtained by the proposed method. According to the figures, the composite array could detect the axial crack effectively. The length and depth of the crack can be estimated from the image as 9 and $1.5 \mathrm{~mm}$, respectively. The width of the first crack was not detected, because there is no effective reflecting interface on the crack for the

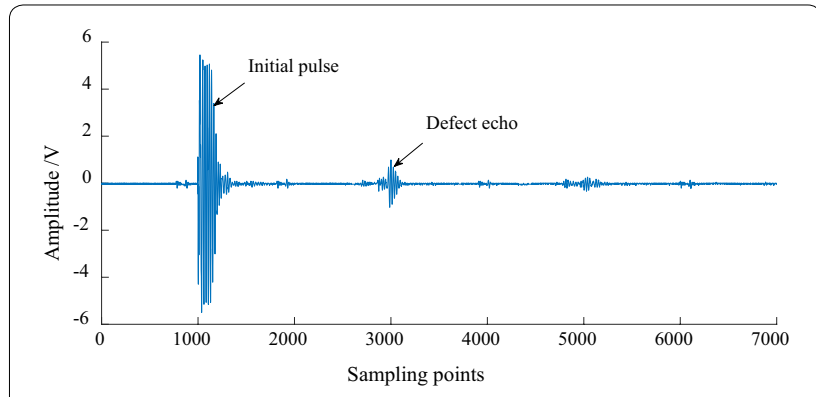

Figure 6 Defect echo signal of the first crack oblique incidence transducers to receive any effective echo signal.

\subsection{Second Crack}

The second crack was an external circumferential crack on the pipeline sample. In the actual inspection, two transducers in the composite array received reflected echoes repeatedly at different stepping positions. Figure $8(a)$ and (b) illustrate one of the received signals from each of those two transducers. Figure 9(a) depicts a picture of the second crack, and Figure 9(b) and (c) present images of the second crack on the pipeline sample. The experimental results showed that the composite array could effectively detect external cracks on the pipeline. The length and depth of the second crack can be estimated from the images as 11.4 and $5.1 \mathrm{~mm}$, respectively. The width of the second crack was not obtained too for the same reason of the first crack.

\subsection{Third Crack}

The third crack was a circumferential crack, which was manufactured on the internal surface of the pipeline sample. As with the inspection of the second crack, two transducers in the composite array received echo signals repeatedly at different stepping positions. Figure 10(a) and (b) separately illustrate one of the echo signals of these two transducers. The ultrasound image of the third crack is depicted in Figure 11; specifically, Figure 11(a) presents the picture of the third crack, and Figure 11(b) and (c) depict the images of this crack projected on the longitudinal section and transverse section of the pipeline sample, respectively. The results showed that this composite array could effectively detect internal circumferential cracks on the pipeline. The estimated length and depth of the third crack were 10.7 and $4.6 \mathrm{~mm}$, respectively. As with the detection of the second crack, no circumferential oblique incidence transducers received any effective echo signal; thus, the width of the third crack was not detected too.

The proposed composite transducer array can detect internal and external cracks with different sizes and orientations in pipelines. The quantization parameters of a defect can be estimated from its ultrasound image. 

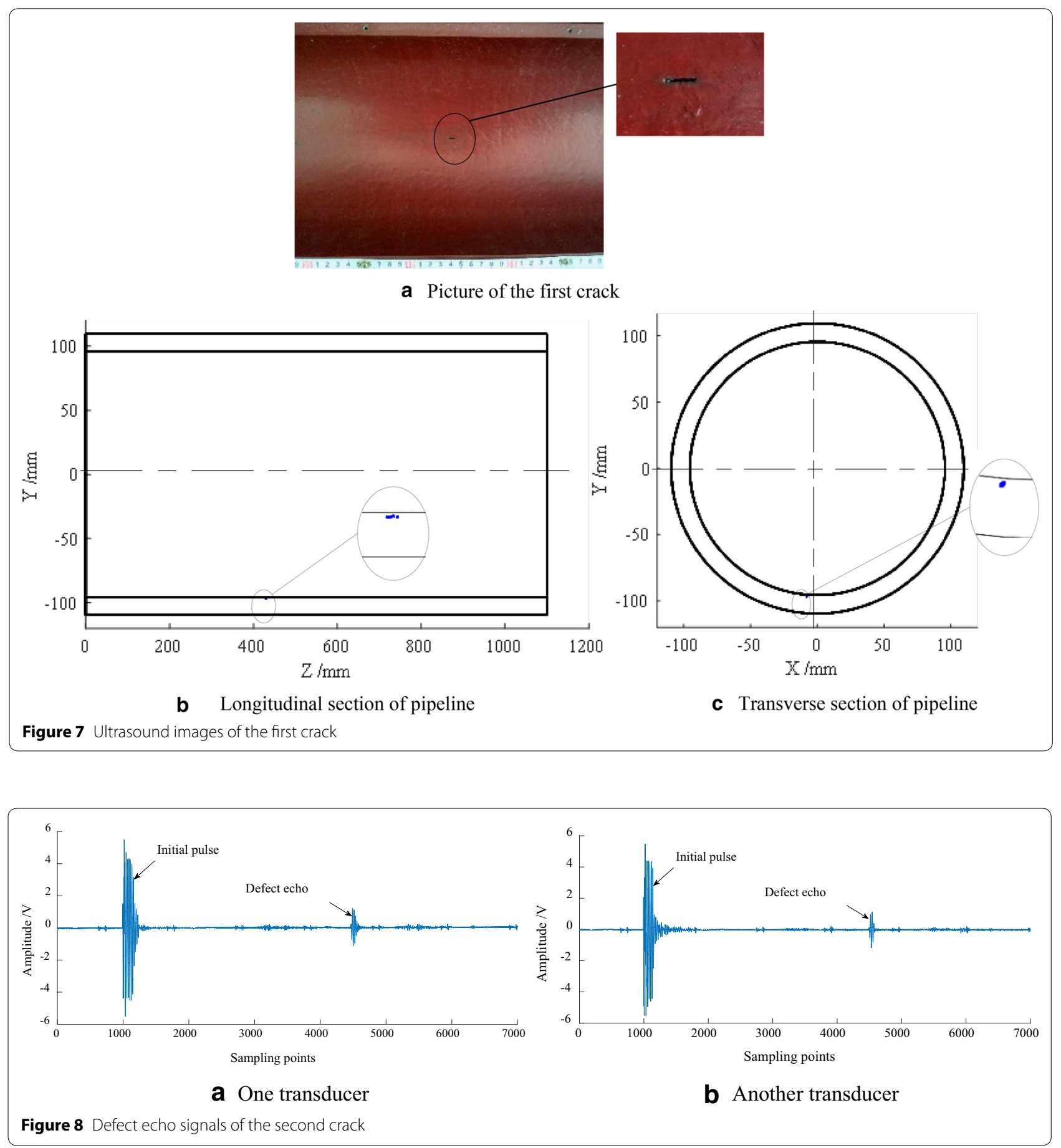

The reasons for the crack parameters estimated from the ultrasonic image differ from the actual crack dimension are as follows.
(1) The accuracy of the crack quantitative parameters estimated from the reflected ultrasonic signal is theoretically not so high. 


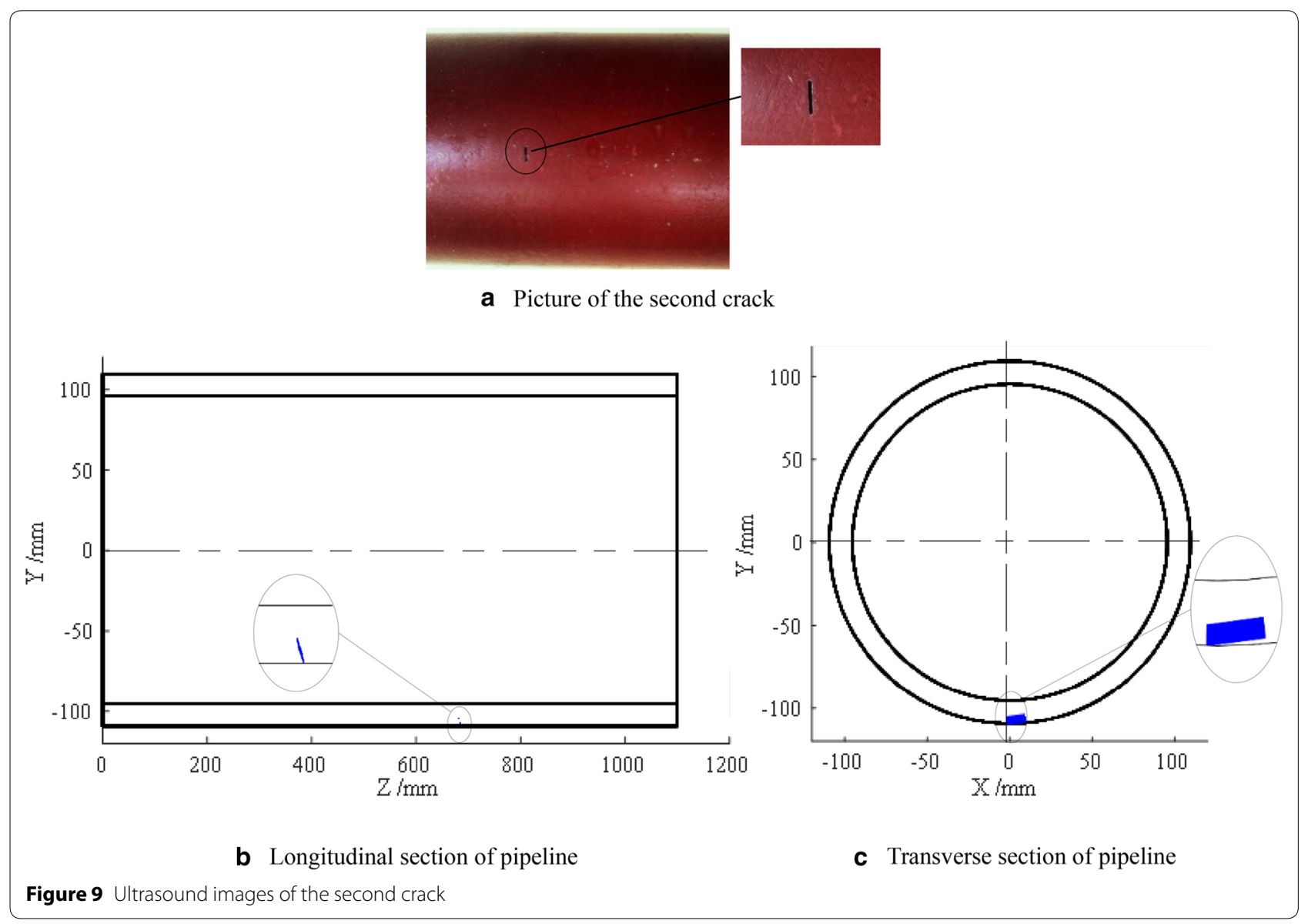

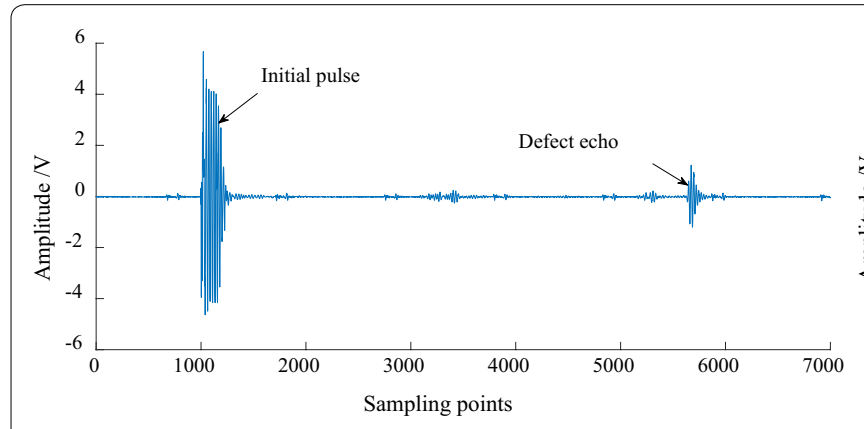

a One transducer

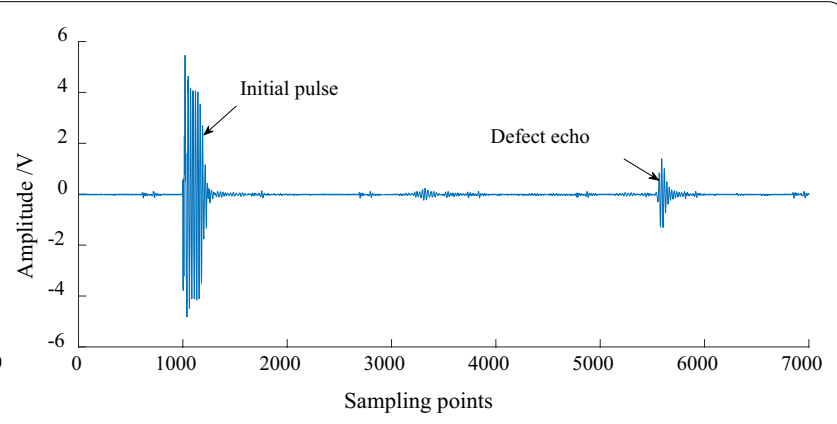

b Another transducer

Figure 10 Defect echo signals of the third crack

(2) The position and size of the crack are estimated by geometrical acoustics theory, due to the spread angle of the acoustic beam, the estimated error is existed.
(3) The step distance of the transducer moving in the pipeline will affect the testing accuracy.

(4) The volume of the cubical element for imaging will affect the accuracy of the crack parameters. 


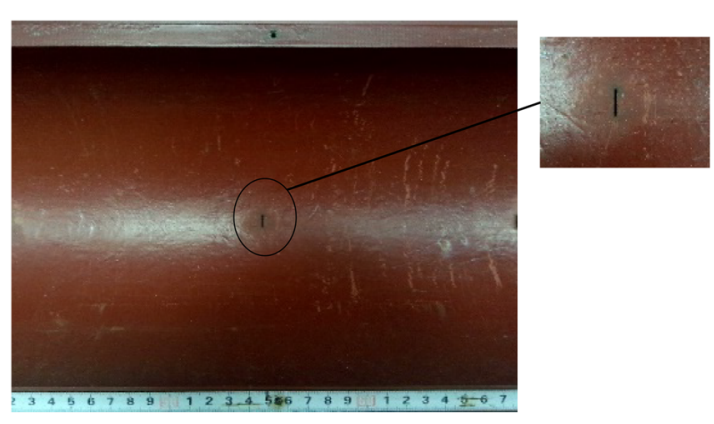

a Picture of the third crack

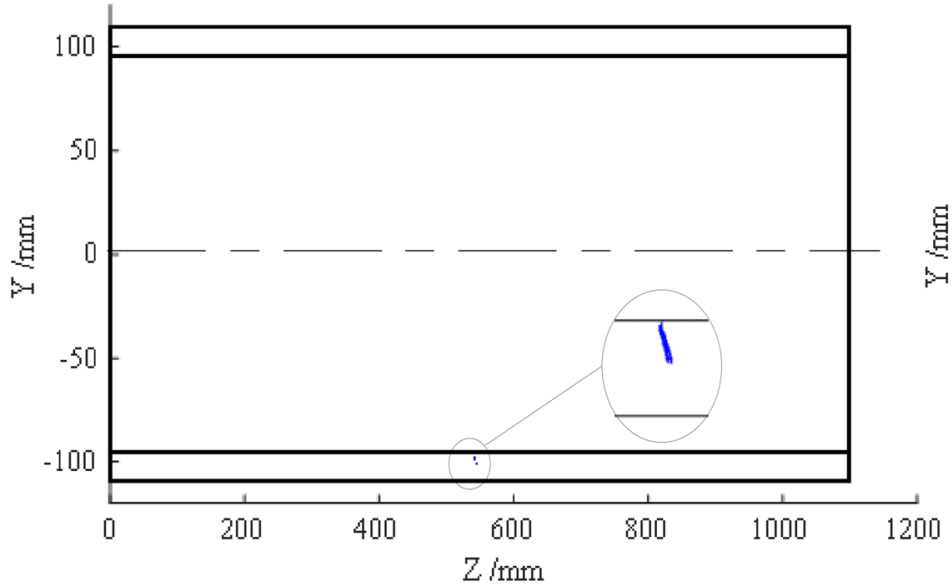

b Longitudinal section of pipeline

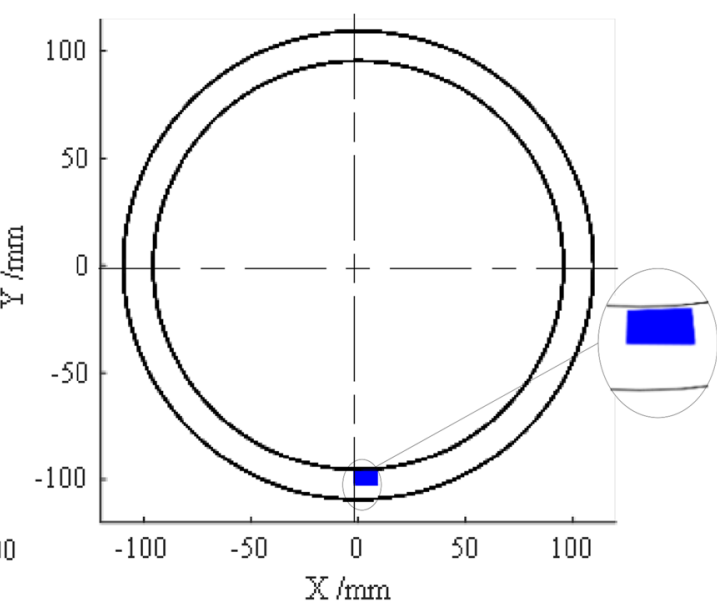

C Transverse section of pipeline

Figure 11 Ultrasound images of the third crack

\section{Conclusions}

(1) To solve the shortcomings of traditional transducer arrays incurrent ultrasonic pipeline inspection, a new composite transducer array is presented, which consists of acoustic beam normal incidence mode, axial oblique incidence mode, and circumferential oblique incidence mode. The three acoustic beam incidence modes are more sensitive to the cracks with various orientations.

(2) An ultrasound imaging method corresponding to the composite array is also proposed. According to the image, the quantization parameters of defects with different sizes and types can be clearly observed, which is helpful for defect evaluation.

(3) The experiments of pipeline ultrasonic testing with three crack types are implemented. The experimental results reveal that the proposed method can acquire holistic images of defects. Information regarding the spatial orientation and distribution of defects can be discovered more conveniently and efficiently with this method than with other commonly used methods, especially for small cracks.
(4) The crack with the dimension of $10 \times 1 \times 3.6 \mathrm{~mm}^{3}$ (length $\times$ width $\times$ depth) is detected in the experiments. Compared to the up-to-date commercial ultrasonic testing equipments for pipeline inner inspection, the proposed technique can detect smaller defects than these testing equipments.

Authors' Contributions

S-PS was in charge of the whole trial; S-PS and Y-JN wrote the manuscript; Y-JN assisted with sampling and laboratory analyses. All authors read and approved the final manuscript.

\section{Authors' Information}

Shou-Peng Song, born in 1967, is currently a professor at Department of Instrument Science and Engineering, School of Mechanical Engineering, Jiangsu University, China. He received his PhD degree from Department of Instrument Science and Technology, Shanghai Jiaotong University, China, in 2006. His research interests include ultrasonic testing, circuit design, signal processing, compressive sensing and signal sparse sampling.

Ying-Jie Ni, born in 1992, is currently working towards the Master of Engineering degree at Department of Instrument Science and Engineering, School of Mechanical Engineering, Jiangsu University, China. His research interests include ultrasound imaging of pipeline defects and parameter estimation of ultrasonic signal from sparse sampling data. 


\section{Competing Interests}

The authors declare that they have no competing interests.

\section{Funding}

Supported by National Natural Science Foundation of China (Grant No. 51375217).

\section{Publisher's Note}

Springer Nature remains neutral with regard to jurisdictional claims in published maps and institutional affiliations.

Received: 6 September 2016 Accepted: 12 September 2018

Published online: 21 September 2018

\section{References}

[1] Y Di, J Shuai, X L Wang, et al. Study on methods for classifying oil \& gas pipeline incidents. China Safety Science Journal, 2013, 23(7): 109-115. (in (hinese).

[2] V Onderka. 2006-2009 triennium work report working committee 2: storage. Proceedings of the 24th World Gas Conference, Buenos Aires, Argentina, 5-9 October, 2009.

[3] J Shuai. American oil and gas pipeline accident and enlightenments. Oil \& Gas Storage and Transportation, 2010, (11): 806-809. (in Chinese)

[4] J X Zhou, X Wu, Z F Guo. The enlightenment of American pipeline accident to Chinese oil and gas pipeline safety. Journal of Safety Science and Technology, 2014, (S1): 73-78. (in Chinese)

[5] W M Wang, X H Wang, S M Zhang, et al. Long-distance pipeline ultrasonic internal inspection-state of the art. Oil \& Gas Storage and Transportation, 2014, 33(1): 5-9. (in Chinese)

[6] S P Song, P W Que, WY Zhang. Design of line-focusing ultrasonic transducer array used for flaw detection in seabed pipelines. Chinese Journal of Scientific Instrument, 2006, 27(5): 547-550. (in Chinese)

[7] A Hugger, D Allen, I Lachtchouk, et al. Ultrasonic phased array crack detection update. Proceedings of the 4th Pipeline Technology Conference 2009, Hannover Messe, Hannover, Germany, 22-23 April, 2009.

[8] Y Zhou, HR Dong, Z G Zhou, et al. The development of oil and gas pipeline inner detection technology. China Petroleum Machinery, 2011, 39(3): 74-77. (in Chinese)

[9] UltraScan Duo detects cracks and metal loss in a single in-line inspection. 2005. http://site.ge-energy.com/prod_serv/serv/pipeline/en/insp_srvcs/ metal_loss/ultrascan_duo/index.htm.
[10] L Huyse, A V Roodselaar, J Onderdonk, et al. Improvements in the accurate estimation of top of the line internal corrosion of subsea pipelines on the basis of in-line inspection data. Proceedings of the 8th International Pipeline Conference, Calgary, AB, Canada, September 27 - October 1, 2010: 1-8.

[11] R Kania, S Klein, J Marr, et al. Validation of EMAT technology for gas pipeline crack inspection. 9th International Pipeline Conference, Volume 2: Pipeline Integrity Management, Alberta, Canada, 24-28 September, 2012: 73-77.

[12] W Gong, RY He, H L Zhao, et al. Cutting-edge applications of foreign in-line inspection technologies to oil and gas pipeline. Pipeline Technique and Equipment, 2013(4): 24-26. (in Chinese)

[13] RoCD EMAT-C service in-line high resolution axial crack detection and sizing 2016. http://www.rosen-group.com/global/solutions/services/service/ rocd-emat-c.html.

[14] THennig, G Lokwani. EVO series 1.0 - latest generation of UT crack and corrosion tools for high speed pipeline inspection. Proceedings of Abu Dhabi International Petroleum Exhibition and Conference, Abu Dhabi, UAE, 9-12 November, 2015.

[15] P Smorscek. NDT global launches ultrasonic inspection tool for crack detection of 6" liquid pipelines. Pipeline Technology Journal, 2014(1): 16. http://www.pipeline-journal.net/journal/archive.

[16] Crack detection brochure English. 2015, http://www.ndt-global.com/ company/latest-news-events/company-news.html.

[17] X J Li, L Wang, X R Gao, et al. Imaging technology of ultrasonic nondestructive detection. Modern Electronics Technique, 2010, (21): 120-122. (in Chinese)

[18] Z H Chen, Y W Shi, H Y Zhao, et al. Nonlinear ultrasonic testing and imaging for tiny flaw. Acta Acustica, 2010, 5(1): 9-13. (in Chinese)

[19] L Satyanarayan, C Sridhar, C V Krishnamurthy, et al. Simulation of ultrasonic phased array technique for imaging and sizing of defects using longitudinal waves. International Journal of Pressure Vessels and Piping, 2007, (84): 716-729.

[20] Z G Zhou, Y Li, W B Zhou. Ultrasonic phased array post-processing imaging techniques: a review. Journal of Mechanical Engineering, 2016, 52(6): 1-11. (in Chinese)

[21] Research on ultrasonic phased array system for automatic defect detection of pipeline girth welds. The 9th International Conference on Electronic Measurement \& Instruments, Beijing, 16-19 August, 2009: 1-903-1-907.

[22] J P Jiao, S F Yang, T Ma, et al. Nonlinear imaging using ultrasonic array under different phased mode. Journal of Mechanical Engineering, 2013, 49(6): 98-98. (in Chinese)

\section{Submit your manuscript to a SpringerOpen ${ }^{\circ}$ journal and benefit from:}

- Convenient online submission

- Rigorous peer review

- Open access: articles freely available online

- High visibility within the field

- Retaining the copyright to your article

Submit your next manuscript at springeropen.com 